\title{
AOR
}

Selected Papers of \#AolR2020: The $21^{\text {st }}$ Annual Conference of the Association of Internet Researchers Virtual Event / 27-31 October 2020

\section{"I'VE GOTTA DO IT FOR THE BIT": MEMETIC MEDIA AND COLLECTIVE IDENTITY THROUGH STREAMER PERFORMANCE ON TWITCH}

Nathan J Jackson

School of the Arts and Media, UNSW Sydney

Videogame livestreamers on the platform Twitch present a carefully curated version of themselves negotiated in part via interactions with their viewers. This persona is encoded not just through their live performance, but also through other platform features including streamer-specific emoticons (emotes) and audio-visual overlays triggered by stream events such as donations and subscriptions (alerts). From these customisable features emerges a complicated feedback loop between the streamer and non-streamer participants that ultimately results in a set of collective values performed and refined by both parties over time. In this paper I interrogate how the incorporation of Internet memes into streaming personas creates accessible avenues for communication with and between members of this collective that contribute significantly to this value system.

Memes operate based on a core duality between commitment to a fixed set of properties and creative variation - this duality has been referred to using several different terminologies (see Wiggins \& Bowers, 2015; Milner, 2016; Phillips \& Milner, 2018). As such, there is a necessary plurality to memes considering their diverse manifestations, as emphasised by Limor Shifman's (2014) definition of the term. This complicates the meaning-making process of memes as they carry not just the intended value of each manifestation's creator, but also the latent values of the meme template and the referent texts.

I define the term memesis - the core concept of this paper - as the cultural process by which Internet users draw upon existing memes in order to create new memetic media. A portmanteau of the words 'meme' and 'mimesis', memesis emphasises the performative process of creating the memetic product. The live performance and interaction with viewers enabled by livestreaming makes Twitch an ideal site for an introduction to this concept. I will show how memesis occurs live on Twitch, where it simultaneously reinforces and redefines aspects of collective identity (in a memetic fashion). In this way, this paper will contribute to conversations around how memes 
influence collective identity and values (Gal, Shifman \& Kampf, 2016; Nissenbaum \& Shifman, 2017).

T.L. Taylor (2018) acknowledges the performativity underpinning successful streaming through connections between users facilitated by language, jokes, and memes. Further, the liveness and direct interaction enabled by the livestreaming mode are key to strengthening these connections (Woodcock and Johnson, 2019). Understanding how memes are deployed in this environment, where for instance there isn't time to prepare an image macro, is essential to examining the labour and creativity involved in stream interactions. In this way, memesis can also be understood as a form of relational labour (Baym, 2015).

Through two case studies, I will discuss how memesis affects streamer agency and impacts the structure and values of stream collectives. The first, RayNarvaezJr, is an American streamer whose identity performance extends to his emotes and Twitch alerts, which explicitly reference his Puerto Rican heritage. He performs memesis through Twitch alerts for donations of memetic amounts, such as US\$69.69 or US\$420, and subscriber emotes, for example brownDAB, which depicts him performing the memetic dab gesture. Members of chat are then able to enact memesis on RayNarvaezJr's behalf and through his persona by engaging with these features.

The second case study, PaladinAmber, is an Australian streamer renowned for her readiness to challenge the hegemonic masculinity of the platform. Her approach towards inappropriate stream behaviour undermines the underlying values motivating the behaviours without alienating other members of her audience (Grayson, 2019). In fact, PaladinAmber contrasts neatly with Mia Consalvo's (2018) analysis of Kaceytron, who tackles similar problematic attitudes through very different means. Further, Kishonna L Gray's (2017) work on deviance suggests that PaladinAmber is doubly resistant through a combination of her marginalised identity on the platform and explicit rejection of the dominant (white male) value system. PaladinAmber's creative use of memetic overlays and multiple cameras is core to her streaming persona. Like RayNarvaezJr, PaladinAmber performs memesis in two stages (preparation and execution), however she maintains control over both by choosing which overlays to implement and when. Her performance becomes more complex when these moments exist in an ambivalent state as both serious confrontation and humorous memesis.

Considering memesis through these case studies draws focus towards how streamers interact with the memescape in order to construct and maintain a streaming persona, as well as create connections with and between their viewers. This paper will touch on a number of key opportunities for analysing stream collectives that emerge through memesis, including: the impact of the streamer's identity on the value system within their stream; a temporal dynamics of values determined by both the rate of change of particular memes and the regularity with which they occur within streams; engagement with memes as active participation in the collective value system and the associated boundary work; and the contrasting explicit and latent values of manifestations of particular memes. Whilst demonstrated within Twitch in this paper, each of these ideas is ripe for exploration within other digital cultural arenas. 
This paper draws upon ongoing ethnographic research as part of a larger project involving the close examination of a small number of Twitch streamers with a focus on streaming personas and the different forms of collective identity that they facilitate. This research is combined here with scholarship on memes in order to examine memesis as a method for the construction and communication of a collective value system. An essential but generally unseen part of meme-making, memesis renders visible the encoding of meaning and value into manifestations of meme, and so this concept names a practice that warrants attention not just on Twitch but within broader digital cultural spheres.

\section{References}

Baym, N. (2015). Connect With Your Audience! The Relational Labor of Connection. The Communication Review 18(1): 14-22.

Consalvo, M. (2018). 'Kaceytron and transgressive play on Twitch.tv', in Jørgensen K. and Karlsen, F. (eds.) Transgressions in Games and Play, Cambridge, MA, MIT Press, pp. 83-98.

Gal, N., Shifman, L and Kampf, Z. (2016). "It Gets Better": Internet memes and the construction of collective identity. New Media \& Society 18:1698-1714.

Gray, K.L. (2017). “'They're just too urban”: Black gamers streaming on Twitch', in Daniels, J., Gregory, K. and Cottom, T.M. (eds.) Digital Sociologies, Bristol, Policy Press, pp. 355-368.

Grayson, N. (2019). Aussie Streamer PaladinAmber Does Not Have Time For Your Shit, Internet Creeps. Retrieved from: https://www.kotaku.com.au/2019/07/aussiestreamer-paladinamber-does-not-have-time-for-your-shit-internet-creeps/. Accessed February 2020.

Milner, R.M. (2016). The World Made Meme: Public Conversations and Participatory Media. MIT Press.

Nissenbaum, A. and Shifman, L. (2017). Internet memes as contested cultural capital: The case of 4chan's /b/board. New Media \& Society 19: 483-501.

Phillips, W. and Milner, R.M. (2018). The Ambivalent Internet: Mischief, Oddity, and Antagonism Online. John Wiley \& Sons.

Shifman, L. (2014). MIT Press Essential Knowledge: Memes in Digital Culture. MIT Press.

Taylor, T.L. (2018). Watch Me Play: Twitch and the Rise of Game Live Streaming. Princeton University Press. 
Wiggins, B.E. and Bowers, G.B. (2015). Memes as genre: A structurational analysis of the memescape. New Media \& Society 17: 1886-1906.

Woodcock, J. and Johnson, M.R. (2019). Live Streamers on Twitch.tv as Social Media Influencers: Changes and Challenges for Strategic Communication. International Journal of Strategic Communication 13: 321-335. 